European Association for the Development of Renewable Energies, Environment and Power Quality
International Conference on Renewable Energies and Power Quality (ICREPQ'09)

Valencia (Spain), 15th to 17 th April, 2009

\title{
Capacitor Switching Techniques
}

\author{
S.J. Kulas \\ Faculty of Electrical Engineering \\ Warsaw University of Technology \\ GG 130, Pl. Politechniki 1, 00-662 Warsaw, Poland \\ Phone number: (48-22) 660-5383, Fax number: (48-22) 625-7524, e-mail: Stanislaw.Kulas@ien.pw.edu.pl
}

\begin{abstract}
Capacitance switching applications involve not only interrupting capacitive currents, but also the energizing of capacitor banks, cables and overhead lines. The interruption of a capacitive current can cause dielectric problems for the switching device. The high inrush currents can cause damage to the capacitors of the capacitors bank and to weld the contacts of the switch together. The problems of the capacitive inrush currents and ways to reduce the magnitude of the inrush current, have been chosen for analysis in this paper.
\end{abstract}

\section{Key words}

Inrush current, capacitor bank, synchronous switching.

\section{Introduction}

Power systems contain lumped capacitors such as capacitor banks for voltage regulation or power factor improvement and capacitors that are part of filter banks to filter out higher harmonics. In addition, cable networks on the distribution level form a mainly capacitive load for the switching devices. Capacitive switching requires special attention because, after current interruption, the capacitive load contains an electrical charge and can cause a dielectric re-ignition of the switching device. When this process repeats, the interruption of capacitive currents causes high over-voltages [1], [3], [7].

The interruption of a capacitive current can cause dielectric problems for the switching device, but when a capacitor bank is taken into service, large inrush currents can flow through the substation and can cause problems for the protection system. During closing a switch or circuit breaker in a dominantly capacitive network with capacitor banks or cable line, represented by its capacitance, the transient voltage oscillates along the line at a relatively low single frequency. It has an amplitude that reaches a peak value approximately equal to twice the value of the system voltage that was present at the instant at which the closure of the circuit took place [2].
The questions of the capacitive inrush currents have been chosen for analysis in this paper.

\section{Inserting Capacitor Banks}

Two cases must be considered when closing a capacitive circuit [5], [6]. The first is illustrated in Figure 1, which shows a single-phase representation with lumped elements of capacitive circuit. Here the switch closes on a single bank whose circuit elements are the inductance of the source $L_{S}$ (resistance of the supply $R_{s} \cong 0$ ), the local inductance in the capacitor's cable $L_{C}\left(L_{S}>L_{C}\right)$ and the capacitance $C$ of the bank. This is termed single bank or isolated bank switching.

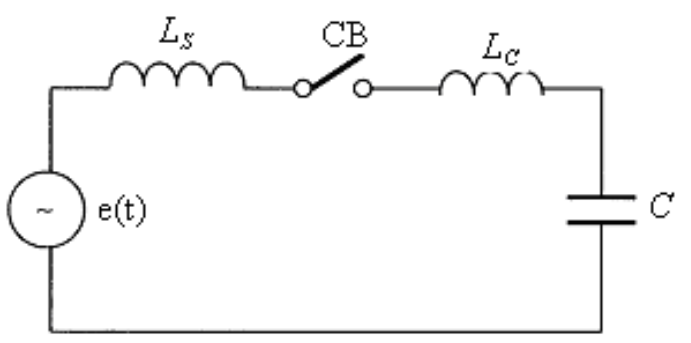

Fig. 1. A single capacitor bank circuit

The transient current $i(t)$ depends on the initial conditions in the circuit and the circuit parameters. If capacitor $C$ is charged to voltage $u_{c 0}$ at the time $t_{0}$, when the switching device closes (Fig. 2), the expression for the current is

$$
i=\left[e\left(t_{0}\right)-u_{c 0}\right] \frac{1}{\sqrt{\frac{L_{S}}{C}}} \sin \omega_{0} t
$$

where:

$$
\omega_{0}=\sqrt{\frac{1}{L_{S} C}}
$$




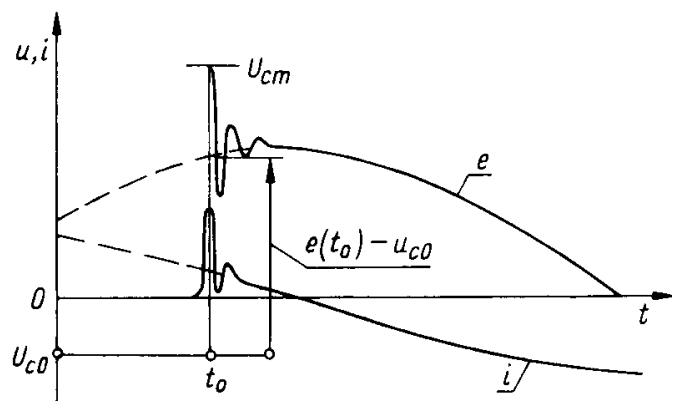

Fig. 2. Illustrating the voltage and current in the capacitor bank circuit

The will be considerable damping in a practical capacitor circuit, so the sinusoidal current will decay rapidly and the circuit current will be established in the capacitor circuit.

The instantaneous voltage of the supplying source at the instant of closing of the switching device at $t_{0}$ is equal $E_{m}$, so the peak of the transient current is given by

$$
i_{\max }=\left(E_{m}-u_{c 0}\right) \sqrt{\frac{C}{L_{s}}}
$$

The source voltage, the circuit inductance, the closing angle and the capacitance determine this inrush current's magnitude. The peak of the transient inrush current is more than ten times the value of the peak of the state capacitive current and a high frequency. These rather high inrush currents can cause damage to the capacitors of the capacitors bank. But the switching device can also be damaged. When a dielectric breakdown occurs just before the contacts touch during the closing operation (a so-called prestrike), the rather large inrush current can cause the contact material to meld. Especially in cases in which vacuum circuit breakers are used to switch the capacitor banks, the contacts might weld together. A prestrike in a minimum-oil circuit breaker or in an $\mathrm{SF}_{6}$ circuit breaker can do other damages to the breaker. The suddenly created plasma channel causes a shock wave and can make, in the case of a minimum-oil circuit breaker, the complete interrupter to explode or, when $\mathrm{SF}_{6}$ is the extinguishing medium, can sometimes damage the arcing chamber or nozzle.

The second case is shown in Figure 3 for switching in another capacitor bank to a capacitor circuit, which is already operational. This is termed back-to-back or bankto-bank capacitor switching.

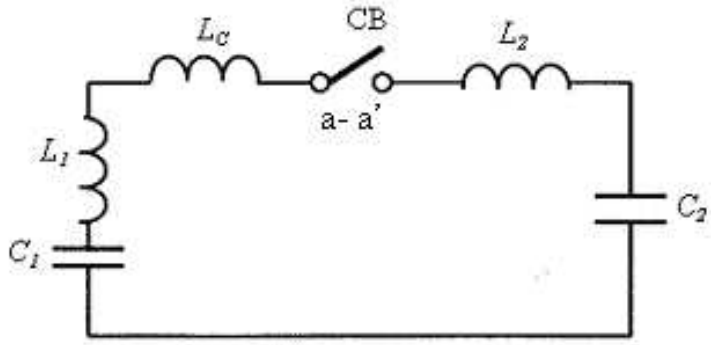

Fig.3. A back-to-back capacitor bank circuit
In this kind of capacitor circuit, only the local capacitor circuits dominate the inrush current to the connecting bus for a few milliseconds. So in Figure 3, the source voltage and the source inductance are not shown.

If during the closing operation, a dielectric breakdown between contacts $a-a^{\prime}$ of a switch occurs at the amplitude $u_{a-a^{\prime}}$ just before the contacts touch during the closing operation, the expression for the current is

$$
i_{2}(t)=u_{a a^{\prime}}(0) \frac{1}{\sqrt{\left(L_{1}+L_{2}\right)\left(\frac{1}{C_{1}}+\frac{1}{C_{2}}\right)}} \sin \omega_{02} t
$$

where:

$$
\begin{gathered}
\omega_{02}=\sqrt{\frac{1}{L_{1}+L_{2}}\left(\frac{1}{C_{1}}+\frac{1}{C_{2}}\right)} \\
u_{a-a^{\prime}}(0)=E_{m} \frac{1}{1-\omega^{2} C_{1}\left(L_{s}+L_{1}\right)} \\
\omega=\sqrt{\frac{1}{L_{s} C}}
\end{gathered}
$$

The transient inrush current can be very high, in the tens of kiloampers range. This current now only stresses the components in the local parallel capacitor circuit and does not affect the rest of the system.

In both cases, the inrush current can result in minor welding of the contact spots during the closing operation when a prestrike arc develops between the closing contacts and during contact bouncing once they touch.

When found that it is necessary to limit the magnitude and frequency of the inrush current, what is recommended is the use of:

1. closing resistor, which is inserted momentarily during the capacitor energizing period and then is subsequently bypassed (Fig. 4).

2.

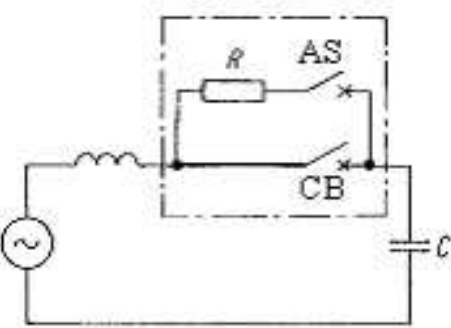

Fig. 4. Circuit breaker CB with resistor $\mathrm{R}$ and auxiliary switch AS

3. By permanently placing a fixed reactance in the capacitor circuit [6]; it is necessary to remember that the reactance will increase energy losses in the system and will also reduce the effectiveness of the capacitors. 
4. By synchronizing the closing of the circuit breaker; the closing of the contacts is synchronized so that it takes place at or very near the zero voltage thus effectively reducing the inrush current [2]. A comparison of the voltage transient for a non-synchronous operation and a synchronized closing is shown in Figure 5a,b.

a)

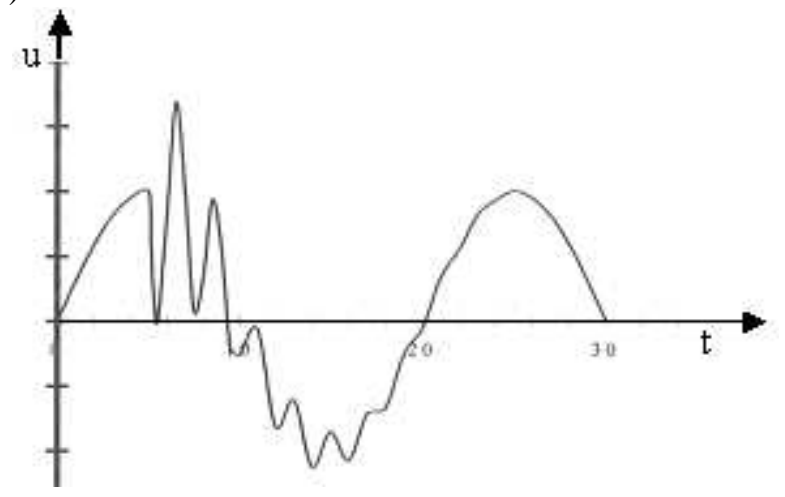

b)

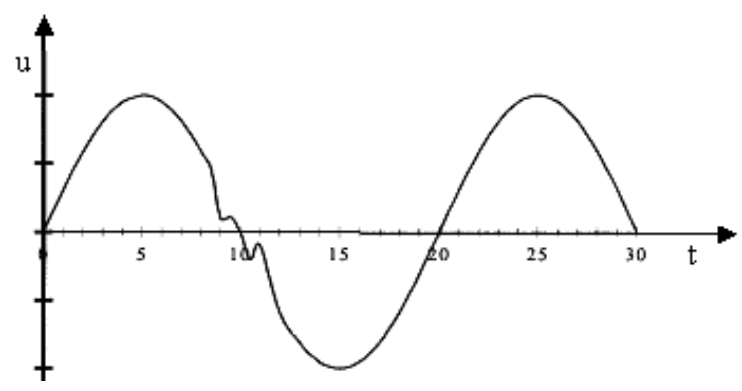

Fig. 5. Voltage corresponding to: a) no-synchronous, b) synchronous, closing into a capacitor bank

As it can be seen in the illustrations the higher frequency component of the voltage is practically eliminated when the contacts are closed at a nominal voltage zero condition.

\section{Synchronous Switching}

In order to completely eliminate the overvoltages and the inrush current produced by the closure of a circuit breaker onto a capacitor bank it is required that there be a zero voltage difference across the contacts of the circuit breaker at the time where the contacts meet. Naturally this is not always possible simply because some deviation from the optimum operating conditions has to be expected [1]-[4].

Consistency in the making times of the circuit breaker is absolutely essential for successful implementation of all types of synchronous switching. However, considering the fact that a circuit breaker is a mechanical device and even though modern designs highly reliable, further improvement still is necessary. It is indispensable to closely analyze the mechanical and electrical properties of the design including contact velocity, contact opening time, contact closing time, minimum arcing time for different interrupting duties and current levels and cold gap voltage withstand capability. Furthermore, and in relation to the operating times, the effects of control voltage fluctuations, ambient temperature, tolerances of the mechanism's stored energy and operating wear must also be considered [3]. Of all the parameters that have been mentioned above the ones that have the greatest influence in the consistent timing of a circuit breaker are the ambient temperature, the level of energy stored in the operating mechanism and the control voltage level.

The break down of the contact gap occurs at the moment of equalising of contact gap dielectric strength $\mathrm{u}_{\mathrm{p}}\left(\mathrm{t}_{\mathrm{s}}, \mathrm{t}\right)$ with the momentary value of the voltage $\mathrm{u}(\mathrm{t})$ applied to the gap (Fig. 6). This is illustrated in the figure where the absolute value of a sinusoidal voltage has been plotted against the slope of an assumed gap voltage withstand characteristic. As it can be seen in the figure the point where the flashover takes place corresponds to the intersection of two curves.

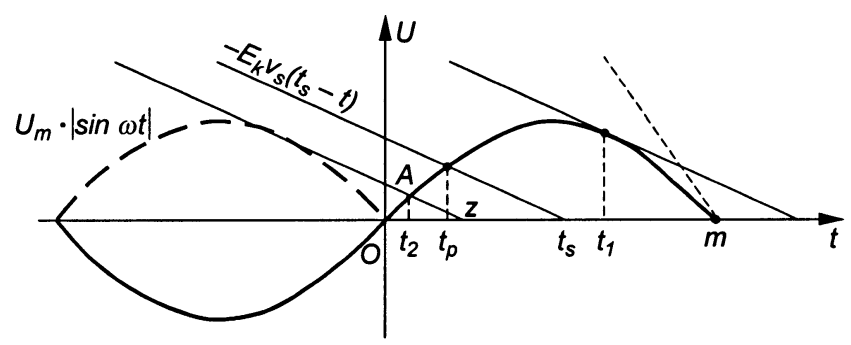

Fig. 6. Relation between system voltage and interrupter gap withstand capability

Assuming that the breakdown voltage is proportional to the distance between the contacts, and that it is not polarity-dependent, it is possible to determine the time $t_{p}$ when the breakdown occurs during current switching on from the relation:

$$
U_{m}|\sin \omega t|=E_{k} v_{s}\left(t_{s}-t_{p}\right)=E_{k} v_{s} t_{a}
$$

where:

$\mathrm{E}_{\mathrm{k}}-$ critical value of electric field strength,

$t_{p}$ - moment of break down of contact gap,

$\mathrm{t}_{\mathrm{s}}$ - moment of contact closing,

$\mathrm{v}_{\mathrm{s}}-$ closing velocity of the contact gap.

The arcing time $t_{a}$ depends on the value of the contact gap withstand and on the value of closing velocity $v_{s}$ of contact. In the given conditions, this time will be the shorter, the closer to zero value crossing point is the voltage phase.

For the sake of switching the current on in an electric circuit at the exact moment of voltage crossing the zero point in order to avoid flashover the contact gap in the process of switching on, two practical conditions must be fulfiled [4]:

1. The dynamic contact gap withstand during switch contact closing should be higher than the momentary value of the supply voltage;

2. The scatter making time $\left(t_{s}-t_{p}\right)$ of electric should be possibly lowest; for making switches and synchronous switching the scatter making time value should be considered satisfactory if: 


$$
\Delta\left(t_{s}-t_{p}\right) \leq 5^{0} e l
$$

Switching current on is hence possible at any voltage phase angle (Fig. 6) including the phase angle corresponding to the moment of voltage passing the zero value, provided that the condition $\mathrm{k}$, is met.

$$
k=\frac{E_{k} v_{s}}{\omega U_{m}} \geq 1
$$

Minimum value of the velocity of contacts at the moment of their mechanical impact, at which the contact gap will not be breakdown during electric circuit switching on, may hence be determined from the relationship:

$$
\left.v_{s}\right\rangle \frac{\omega U_{m}}{n E_{k}}
$$

The selection of a determined phase of switching current on requires hence, the application of an electronic system to control the process of the switches, e. g. making switch or synchronous switch.

\section{Conclusion}

The magnitude and frequency of the inrush current resulting from energezing capacitor bank is a function of:

- the point on the wave of the applied voltage where the contacts were closed,

- the capacitance and inductance of the circuit,

- the charge on the capacitor at closing time

- any damping resistance contained in the circuit,

Synchronous switching, both opening and closing, of short circuit currents is a desirable feature from the point of view of reducing contact erosion. The reduction of the contact wear directly translates into an extension of the usable operating life of the circuit breaker.

For capacitance switching, failures are often reported which are the direct result of inrush currents and overvoltages that have propagated themselves into lower voltage networks causing damage especially to electronic equipment connected to the circuit.

\section{Acknowledgments}

The authors acknowledge to Polish State Committee for Scientific Research the contribution of this work financed as granted research project No. N510 005 32/0359.

\section{References}

[1] Z. Ciok, Non-Simultaneous short-circuits in three phase networks with special consideration to the effect upon the operation of circuit-breakers, CIGRE Session 1962, report 120.

[2] R.D. Garzon, High voltage circuit breakers, Marcel Dekker, Inc., New York - Basel, 2002.

[3] A. Khan, D.S. Johnson, J.R. Meyer, K.B. Hapke, Development of new synchronous closing circuit breaker for shunt capacitor bank energization, SixtyFirst Annual International Conference of Doble Clients, Berlin, Paper 5E, 1994.

[4] S.J. Kulas, The influence of contact closing velocity on arc duration, Proceedings of the $10^{\text {th }}$ International Conference SAP 2005 on, Switching Arc Phenomena, pp. 106-110, Łódź, Poland, 2005.

[5] J. Maksymiuk, Electrical Apparatuses, WNT Warsaw, Poland (in polish).

[6] P.G. Slade, The vacuum interrupter, CRC Press, New York 2008.

[7] Ware B., Reckleff. J., Mauthe G., Schett G., Synchronous Switching of Power Systems, CIGRE Session 1990, Report No. 13-205. 